\title{
Using AGN Variability Surveys to explore the AGN-Galaxy Connection
}

\author{
Vicki L. Sarajedini \\ Department of Astronomy, University of Florida, \\ 211 Bryant Space Science Center, Gainesville, FL USA \\ email: vicsaraj@ufl.edu
}

\begin{abstract}
Variability is a successful technique used to identify active galactic nuclei in both ground and space-based galaxy surveys. Optical variability surveys using HST have uncovered a number of AGN in deep extragalactic fields extending to $\mathrm{z} \sim 3$ and probing intrinsically faint sources. Mid-IR variability surveys using Spitzer have identified a significant number of AGN and are particularly sensitive to obscured sources. Many variability-detected AGN are not strong X-ray sources or lack the characteristic colors of AGN and would thus be unidentified using other selection techniques. In this conference proceedings, I discuss the nature of the variable sources and their host galaxies identified in deep extragalactic optical and mid-IR surveys.
\end{abstract}

Keywords. galaxies:active, galaxies: evolution, surveys

\section{Introduction}

Active Galactic Nuclei (AGNs) are galaxies currently accreting significant amounts of material onto their central super massive black holes (SMBHs). These galaxies were once thought to be oddities among the total galaxy population but are now seen as important mile markers on the broad road of galaxy evolution. SMBHs are now believed to exist in the centers of all galaxies containing a significant bulge component (Kormendy \& Richstone 1995), and an observed relation between the mass of the SMBH and the bulge stellar mass suggests a coupled assembly history between the two (Ferrarese \& Merritt 2000; Gebhardt et al. 2000). AGN may represent an evolutionary phase for many galaxies and could have a significant impact on the star formation history. To understand the role played by AGN in the evolution of galaxies, it is necessary to identify complete samples in galaxy surveys out to redshifts of $\mathrm{z} \sim 3$.

AGN can be identified using a number of techniques such as UV/optical/IR color selection, spectroscopic signatures, X-ray emission, radio emission and variability over a range of timescales. Many of these techniques, however, produce samples biased against galaxies where the host galaxy light dominates that of the AGN. Additionally, surveys in the optical/UV are biased against obscured, dusty AGN/host galaxies. In order to identify largely complete samples of AGN, it is clear that a variety of techniques and selection methods should be employed. There is increasing evidence that AGN selected using different techniques represent separate populations experiencing different phases in AGN/galaxy evolution (e.g. Hickox et al. 2009). In particular, obscured AGN may hold the key to uncovering the primary fueling mechanism for SMBHs (e.g. Hopkins et al. 2007). Samples of AGN with fewer observational biases allow us to better interpret the role AGN play within their host galaxies across cosmic time.

AGN are known variable sources, with almost all AGN selected through other means (e.g. spectroscopic, color selection, UV excess) displaying significant optical variability when observed over several years (Schmidt et al. 2010). The optical/IR flux changes occur 
on timescales of months to years and have been well fit with damped random walk models indicating a characteristic timescale for variability and maximum amplitude at long time intervals (MacLeod et al. 2010). Variability in AGN is likely due to instabilities in the accretion disk (e.g. Li \& Cao 2008) and has been found to correlate with several AGN parameters such as bolometric luminosity, wavelength and black hole mass (MacLeod et al. 2010; Zuo et al. 2012). In addition, intrinsically fainter AGN are found to have a higher amplitude of variability (Trevese et al. 1994; Wilhite et al. 2008) making variability selection particularly well-suited for identifying faint AGN.

There are several advantages to identifying AGN using variability surveys. First, variability detection can be done with two or more images of the same field and is therefore observationally less time consuming than spectroscopic surveys. If the images are of high enough resolution to resolve the nuclear light within the host galaxy, variability surveys can be used to identify intrinsically faint AGN residing in brighter host galaxies. Variability surveys can also be carried out in synergy with other transient source surveys such as the detection of supernovae in distant galaxies. Finally, in the mid-IR, variability surveys are sensitive to dusty/obscured AGN that may be missed at other wavelengths.

\section{Optical Variability Surveys for Faint AGN}

One of the first studies to identify faint, extended sources was conducted by Bershady, Trevese \& Kron (1998), who identified 14 variable galaxies at $\mathrm{z} \sim 0.3$ in their QSO variability survey of Selected Area 57. These sources were found to have generally blue colors and compact nuclei. The three brightest revealed broad emission lines in their spectra with a fourth appearing to be a narrow-line AGN. The others were too faint to obtain spectroscopic confirmation, but it was clear from these results that the surface density of AGN was much higher than previously thought based on the results of spectroscopic and color-selected surveys alone.

This result prompted us to look for varying nuclei in more distant galaxies imaged with HST. The Hubble Deep Field North (HDFN; Williams et al. 1996) was first observed in 1995 and then again in 1997 and 2000. We compared deep ( 100,000 s), V-band images of galaxies in this field over a 5 year period to detect variability within 0.15 " apertures centered at the galactic nucleus. We found 16 galaxies with significantly varying nuclei, indicating an AGN surface density 10 times greater than that determined from other optical detection techniques (Sarajedini, Gilliland \& Kasm 2003). Most of these AGN were too faint for spectroscopic confirmation, with only three revealing spectroscopic signatures of AGN. However, $44 \%$ were detected in the $2 \mathrm{Ms}$ Chandra X-ray survey of the HDFN. These variable nuclei had typical redshifts around $\mathrm{z} \sim 1$ and reached absolute magnitudes of $\mathrm{M}_{B} \sim-17$.

Other programs to identify varying galaxies have been conducted using ground-based multi-epoch imaging surveys. Trevese et al. (2008) and Boutsia et al. (2009) identified 132 optical variables in the ESO 2.2m survey of the Extended Chandra Deep Field South (ECDFS) and found that $75 \%$ of the variables were detected in the $2 \mathrm{Ms}$ X-ray survey of this field. Of the brighter sources with spectroscopic observations, $60 \%$ revealed broad emission lines and $26 \%$ were narrow-line AGN. Since these AGN were identified in groundbased surveys, it is not surprising that a larger fraction of sources are spectroscopically confirmed AGN compared to the HDFN survey since the AGN component must be a larger fraction of the total galaxy+AGN light in order to be identified as varying. This survey revealed that many of the X-ray detected variable AGN displaying narrow emission lines had particularly low X-ray-to-optical flux ratios compared to the broadline AGN. Morokuma et al. (2008) identified 200 optical variables in the Subaru X-ray 
Deep Survey (SXDS) and found that $60 \%$ of the variables were not detected in X-rays. Further analysis of these sources revealed that a significant fraction of the non-X-ray detected variables appeared to be accreting at low-Eddington ratios. The results of these ground-based surveys confirm that optical variability identifies large numbers of AGN not detected in X-ray surveys or that have low X-ray fluxes and that these sources may have different accretion properties than X-ray detected AGN.

A larger HST variability survey was conducted within The Great Observatories Origins Deep Survey fields (GOODS; Giavalisco et a. 2004). These data are comprised of two fields with 5 epochs of imaging (each $\sim 1000 \mathrm{~s}$ deep) obtained over a 6 month time interval. The GOODS survey covers a region of the sky 30 times larger than the HDFN though with shallower epochs. Using 0.15 " diameter apertures to probe the nuclei of galaxies identified in these fields, we found 85 displaying significant variability, representing about $2 \%$ of all galaxies (Sarajedini et al. 2011). Forty-eight percent of the variables were identified in deep Chandra surveys covering these fields, consistent with previous HST and ground-based results. Using photometric redshifts for galaxies in GOODS, we found the variability-selected AGN candidates had intrinsically fainter absolute magnitudes than the X-ray detected AGN in these fields and that optical variability was able to identify AGN to redshifts beyond $\mathrm{z} \sim 3$. Most of the variable nuclei were fainter than $\mathrm{M}_{V}=-22$ with several reaching absolute magnitudes of -18 .

We examined the host galaxy properties of AGN to compare those identified in the GOODS fields via optical variability and X-ray detection. Most X-ray selected AGN lie in the "green valley" of the galaxy color-magnitude diagram, between the blue, star-forming galaxies and those that occupy the red sequence indicating galaxies that have stopped forming stars and consist of older stellar populations. This has been previously observed for X-ray detected AGN and cited as possible evidence that AGN quench star formation in their host galaxies (e.g. Nandra et al. 2007). While many non-X-ray detected variables are found in the green valley, several have bluer colors and fainter overall magnitudes. Based on the derived bolometric luminosities, $\mathrm{BH}$ masses and Eddington ratios for the variability-selected AGN in the GOODS fields, we find that non-X-ray detected variables generally have lower luminosities than X-ray detected variables, but similar BH masses and Eddington ratios. Thus, some of the variables have characteristics like other X-ray selected samples, revealing significant bulges and the possible onset of quenched star formation and decreased $\mathrm{BH}$ accretion rates, while others may be intermediate mass systems still forming stars and accreting at a continuous lower-level, perhaps through stellar winds or bars.

The spectral energy distributions of the variability-selected AGN were studied in Villforth, Sarajedini \& Koekemoer (2012). In this study, SEDs were fit to the fluxes measured at wavelengths extending from the X-ray $(0.2$ to $8 \mathrm{keV})$ through the optical and infrared to the radio $(6 \mathrm{~cm}$ and $20 \mathrm{~cm}$ ). Sixty-one of the variables in the GOODS South field were used for this study and their SEDs were fit with a combination of galaxy and AGN spectral templates. Of those, 43 were well fit with 12 failed fits and 6 false positives (i.e. no AGN component was required to model the SED). This indicates a high confirmation rate $(\sim 90 \%)$ for the variability-selected AGN. About one quarter of the X-ray and nonX-ray detected variables had SEDs that were dominated by the AGN component (more than $70 \%$ AGN). Non-X-ray detected variables had a larger fraction of galaxy-dominated SEDs $(38 \%)$ compared to X-ray detected variables $(24 \%)$. We also found that the current star formation in the host galaxies of AGN appears most prominent in the highest luminosity galaxies, while lower luminosity galaxies appear quiescent or moderately star 


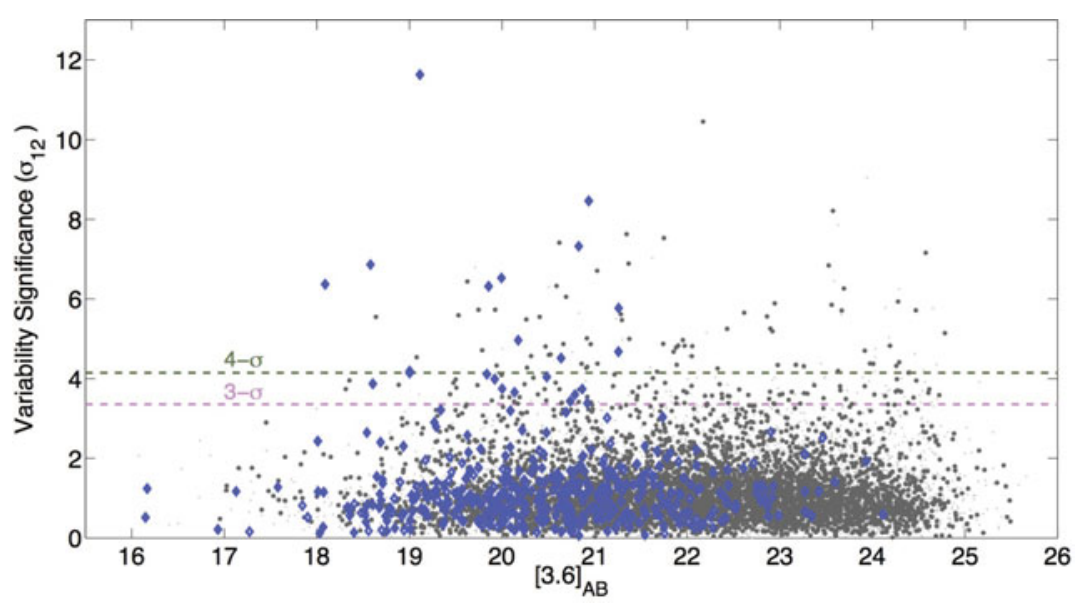

Figure 1. Correlated mid-IR variability significance vs. IR magnitude for sources in the EGS field based on 3 epochs. Dashed lines represent the 3 and $4 \sigma$ variability thresholds. X-ray sources are indicated with blue diamonds.

forming. The overall results indicate that a large portion of the variability-detected AGN would be missed in X-ray and color-selected samples due to the galaxy dominated SEDs.

\section{Mid-IR Variability Surveys for AGN}

In the infrared, Seyferts and QSOs have revealed significant variability with the variations following those in the visible with some delay (Glass 2004, Honig \& Kishimoto 2011). The lags appear consistent with thermal reprocessing of higher energy radiation via dust in the vicinity of the SMBH. This would imply that infrared variability selection could be more sensitive than optical variability or even X-ray emission in the identification of certain types of AGN (e.g. X-ray weak or obscured/Compton thick AGN). These highly obscured sources may also hide key phases of $\mathrm{BH}$ growth including evidence of mergers and asymmetries (e.g. Hopkins et al. 2007).

The Spitzer Extended Deep Survey (SEDS; Ashby et al. 2013) has obtained multiepoch 3.6 and 4.5 micron images of 3 to 4 hour depth per epoch over time intervals of $\sim 1.5$ years in 5 extragalactic fields. We have completed the variability analysis in the Extended Groth Strip (EGS) for the $~ 8,000$ sources detected in the 3.6 micron image reaching depths of 24 th magnitude $(\mathrm{AB})$. The variability analysis reveals $\sim 1 \%$ of galaxies displaying significant $(>4 \sigma)$ variability correlated in the 3.6 and 4.5 micron images (Figure 1 ). We find that $\sim 85 \%$ of the mid-IR variables are not X-ray detected which further illustrates that different AGN identification techniques are sensitive to different AGN samples and populations. Based on the results from this initial field, we estimate that $\sim 400$ variable sources will be identified in the total SEDS survey area.

We matched all galaxies in the SEDS 3.6 micron image with the Rainbow Cosmological Surveys database for the EGS field to obtain photometric redshifts, masses and multiband colors for these galaxies (Perez-Gonzalez et al. 2008). The mid-IR variable AGN candidates are found in galaxies with a broad range of masses $\left(10^{8}-10^{1} 1 \mathrm{M}_{\text {sun }}\right)$ compared to X-ray selected AGN which are mainly found among the higher mass galaxies. The variables have typical redshifts of $\mathrm{z} \sim 1.3$ with some extending to $\mathrm{z} \sim 4$. The rest frame $U-V$ colors of the mid-IR variable host galaxies are distributed through the "green valley" and, similar to the optical variables, several lie in the blue, star-forming region of the galaxy color-magnitude diagram. 


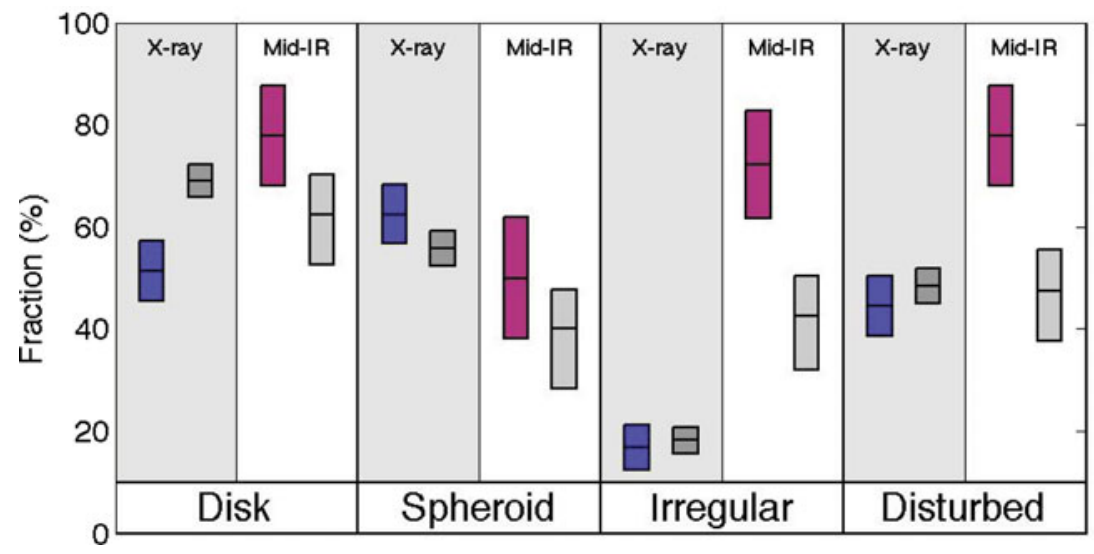

Figure 2. Percentage of galaxies assigned to different morphological and disturbance classes based on visual classification. The blue bars represent AGN identified through X-ray emission (shaded regions; Kocevski et al. 2012) while the magenta bars represent mid-IR variability selected AGN (non-shaded regions) compared to a control sample of galaxies with the same mass distribution for each sample (gray bars in each section). While overall morphologies of the hosts are largely similar, mid-IR variability detected AGN are more likely to reside in irregular/disturbed host galaxies than the control group contrasting with the X-ray detected AGN sample.

We examine the host galaxy morphologies of mid-IR variability-selected AGN to look for evidence of recent merger events or enhanced morphological irregularities. Galaxy mergers have long been suspected as responsible for triggering accretion onto SMBHs. However, several studies have shown little to no evidence of enhanced asymmetry or signs of interactions among AGN host galaxies over normal galaxies (e.g. Grogin et al. 2005). The SEDS fields are also covered by CANDELS (Cosmic Assembly Near-Infrared Deep Extragalactic Legacy Survey; Grogin et al. 2011), which has obtained deep, highresolution HST imaging at 1 to 1.6 microns with HST WFC3 in portions of these fields. Recent CANDELS results have found that, for X-ray selected AGN, the fraction of irregular and disturbed host galaxies is no greater than that of a control sample (Kocevski et al. 2012). In contrast to the X-ray selected sample, our SEDS variability survey finds a significant number of mid-IR variability-selected AGN in disturbed galaxy hosts or those having irregular morphology compared to a control sample of galaxies at similar mass and redshift (Figure 2). These results are based on a sample of $\sim 20$ mid-IR variability-selected AGN in the EGS field that fall within the current coverage of the CANDELS images. The visual analysis was done in the same way as that of Kocevski et al. (2012) but our initial sample covers a much broader redshift and mass range and contains fewer sources. Our control group was chosen to reflect the same demographics as our AGN sample for proper comparison. These results suggest that X-ray surveys miss many highly obscured or X-ray weak AGN and these reveal signatures of recent mergers or galaxy disturbances.

\section{Conclusions}

The results of these and other studies show that AGN can be detected in large numbers through variability surveys probing high redshifts $(\mathrm{z} \sim 3)$ and faint $\left(\mathrm{M}_{B} \sim 18\right)$ luminosities in optical and IR images. Between $1 \%$ and $4 \%$ of galaxy nuclei display optical/IR variability depending on photometric depth, image wavelength and repeatability of the survey epochs. Fifty to eighty percent of the variables are not identified through other AGN detection methods, expanding and complementing multi-wavelength surveys 
for AGN. The spectral energy distributions of many variability-selected AGN are dominated by the host galaxy light with some having AGN components contributing as little as $10 \%$ of the overall flux. Thus, variability is an important tool to uncover otherwise missed AGN.

Recent results from our deep mid-IR variability survey with Spitzer has identified several AGN that lack X-ray emission. Some of these may be Compton thick, obscured sources while others may be intrinsically weak X-ray emitters. The host galaxies of these AGN have morphologies that suggest recent merger activity or galaxy interactions when compared to a control sample of galaxies, in contrast to the morphologies of X-ray selected AGN hosts. These results suggest that mid-IR variability could be an important tool to probe key phases of AGN evolution such as the recent triggering of AGN accretion through mergers or galaxy disturbances.

The results from variability surveys are especially promising in light of future planned multi-epoch observing programs like that for the LSST. Observations with longer and better sampled light curves will produce more robust and complete variability-selected AGN samples. Together with multi-wavelength surveys using a variety of detection techniques, the AGN population can be more powerfully probed and understood in the context of galaxy evolution.

\section{References}

Ashby, M., et al. 2013, ApJ, 769, 80

Bershady, M. A., Trevese, D., \& Kron, R. G. 1998, ApJ, 505, 50

Boutsia, K., Leibundgut, B., Trevese, D., \& Vagnetti, F. 2009. A\&SA, 497, 81

Ferrarese, L. \& Merritt, D. 2000, ApJL, 539, 9

Gebhardt, K., et al. 2000, ApJL, 539, 13

Giavalisco et al. 2004, ApJL, 600, 93

Glass, I. S. 2004, MNRAS, 350, 1049

Grogin, N. A., et al. 2005, ApJ, 627, 97

Grogin, N. A., et al. 2011, ApJS, 197, 35

Hickox, R. C., et al. 2009, ApJ, 696, 891

Honig, S. F. \& Kishimoto, M. 2011, A\& A, 534, 121

Hopkins, P. F., Bundy, K., Hernquist, L., \& Ellis, R. S. 2007, ApJ, 659, 976

Kocevski, D. D. et al. 2012, ApJ, 744, 148

Kormendy, J. \& Richstone, D. 1995, ARA\&A, 33, 581

Li, S-L. \& Cao, X. 2008, MNRAS, 387,41

MacLeod, C. L., et al. 2010, ApJ, 721, 1014

Morokuma, T., et al. 2008, ApJ, 676, 121

Nandra, K. et al. 2007, ApJL, 660, 11

Perez-Gonzalez P. G., Rieke, G. H. \& Villar, V., et al. 2008, ApJ, 675, 234

Sarajedini, V. L., Koo, D. C., Klesman, A., Laird, E., Perez-Gonzalez, P. G., \& Mozena, M. 2011, ApJ, 731, 97

Sarajedini, V. L., Gilliland, R. L., \& Kasm, C. 2003, ApJ, 599, 173

Schmidt, K. B., Marshall, P. J., Rix, H-W., Jester, S., Hennawi, J. F., \& Dobler, G. 2010, ApJ, 714,1194

Trevese, D., Kron, R. G., Majewski, S. R., \& Koo, D. C. 1994, ApJ, 433, 494

Trevese, D., Boutsia, K., Vagnetti, F., Cappellaro, E., \& Puccetti, S. 2008, A\& A, 488, 73

Villforth, C., Sarajedini, V., \& Koekemoer, A. 2012, MNRAS, 426, 360

Wilhite, B. C., Brunner, R. J., Grier, C. J., Schneider, D. P., \& Vanden Berk, D. E. 2008, MNRAS, 283, 1232

Williams, R. E., et al. 1996, AJ, 112, 1335

Zuo, W., Wu, X-B., Liu, Y-Q., \& Jiao, C-L. 2012, ApJ, 758, 104 